\title{
One-Stage Urethroplasty for Strictures at a Rural Hospital
}

\author{
Winston Makanga, ${ }^{1}$ Christian A Agbo ${ }^{2}$ \\ ${ }^{1}$ St Mary's Mission Hospital, Kenya \\ 2 Jos University Teaching Hospital, Nigeria
}

Correspondence to: Dr. Winston Makanga, PO Box 1698, Embu, Kenya; email: wmakanga@yahoo.com

\begin{abstract}
Introduction: St Mary's Mission Hospital manages many inflammatory and traumatic urethral strictures. Previously, we treated strictures with dilatation, but high recurrence and poor patient satisfaction necessitated adoption of reconstructive procedures since 2017. Objective: To review the scope, outcome and complications of urethroplasties using data collected prospectively. Methods: All cases of one-stage urethroplasty were included. Patient biodata and pre-operative adverse factors were collected and analyzed. Results: 23 male patients ranging in age from 24 to 74 years were studied: 9 strictures were inflammatory $(40 \%)$, 9 were traumatic (40\%), 3 (20\%) were recurrent. Nineteen strictures were in the bulbar urethra (83\%), 2 were cases of penile strictures and 1 case each of pan-urethral stricture and pelvic floor urethral distraction defect. Of the 23 procedures, 13 were simple anastomosis (57\%), 5 were dorsal buccal mucosa graft $(\mathrm{BMG})$ urethroplasty $(22 \%), 2$ were cases of non-transecting anastomotic urethroplasty, and 1 case each of ventral BMG urethroplasty and Johansson's and
\end{abstract}

Kulkarni's panurethroplasty. The overall complication rate was $40 \%$ (9 patients). Four patients (17\%) had recurrence; 2 had fistula and 1 case each of persistent UTI, erectile dysfunction and periurethral abscess. Three of the four recurrences had undergone BMG urethroplasty. All cases of simple anastomosis had no recurrence. Conclusion: Our centre has embraced diverse urethroplasties for a wide scope of patients. This study found a significant complication rate for substitution urethroplasties, suggesting a need for careful patient selection and an improvement in technique.

Key words: Stricture, Urethroplasty, One-stage, Complications, Outcome

Ann Afr Surg. 2019; 16(1):16-19

DOI: http://dx.doi.org/10.4314/aas.v16il.4

\section{Conflicts of Interest: None}

\section{Funding: None}

(C) 2019 Author. This work is licensed under the Creative Commons Attribution 4.0 International License.

\section{Introduction}

Urethral stricture is a progressive narrowing or occlusion, or loss of distensibility of any part of the urethra secondary to periurethral spongiofibrosis (1). The cause is either infective or trauma (2). Previous transurethral endoscopic surgery is now a major cause of post-traumatic strictures with a pooled range of $2.2-11.6 \%(3,4)$. Postgonococcal urethritis with complicating strictures has declined with the advent of appropriate antibiotics (5-7).

Urethral bouginage/sounding has historically been used to treat these strictures, partly because of its low cost and the ease of carrying out the procedure. However, these measures are short term at best, and at worst worsen the strictures in both length and breadth (8). Urethroplasty is the only potentially curative modality. These methods are offered only by specialized urethral surgeons. In Kenya, such specialists are few and are found mainly in large referral hospitals located in the main cities $(9,10)$.

The two main types of reconstruction are anastomotic and substitution; both aim to produce a high-caliber, stable and compliant urethra while resolving obstructive symptoms and related sequelae (11). Anastomotic reconstructions rely on apposition of well-mobilized, tension-free urethral ends. Substitution techniques rely on introduction of either free tissue (buccal mucosa) or of flaps obtained from elsewhere, to bridge urethral defects. Most flap techniques are complex with a steep learning curve, and are mostly two-stage procedures. Such complex procedures increase the possibility of complications. Two-stage procedures require close follow-up and introduce a burden to the patient of taking care of the initial surgical site. A second admission and surgery also increase the overall cost. Techniques such as the two-stage Bracka urethroplasty have potentially poor outcomes because the initial buccal mucosal graft shrinks and becomes desiccated by the time 6 months elapse in time for the second-stage surgery. One-stage repair was 
historically suited for solitary, short (sub-centimeter) or multiple contiguous strictures. The indications have now been broadened to include longer strictures including panurethral strictures (12). One-stage procedures offer the benefits of reducing overall cost, better tolerance and reducing the general risk of anesthesia and surgery.

Urethral stricture constitutes significant morbidity at St Mary's Hospital, Kenya. A large number of our patients come from Narok, a region with a high number of postgonococcal urethritis and urethral stricture. These patients present with obstructive lower urinary symptoms, recurrent urinary tract infection, periurethral abscess and/or watering can phenomenon. At St Mary's Hospital, obstructing symptoms have in the past been resolved through dilatation (metal sounding). The use of this technique was occasioned by the cost implication, the lack of a reconstructive urologist and rapid symptomatic relief of the intervention. Recurrence of symptoms has however been unacceptably high, ranging from as short as 1 month to 3 months. This recurrence could be attributed to the complex nature of some of these inflammatory strictures. A patient also has a likelihood of requiring this procedure for life.

We started carrying out urethroplasty in 2017 as an intervention with the potential to cure stricture disease and reduce physical deformity, prolonged follow-up and repeat procedures. This informed our decision to adopt onestage urethroplasty as the procedure of choice. We audited our safety procedures and outcomes at this early stage to determine if our interventions were within international standards.

\section{Methods}

All patients who had undergone one-stage urethroplasty were entered into the study. One-stage urethroplasty is a reconstructive procedure for primary or recurrent urethral stricture that aims to achieve urethral continuity and patency without requiring a second procedure. It includes simple anastomotic urethroplasty, non-transecting anastomotic urethroplasty, buccal mucosal graft (ventral, dorsal and long dorsal panurethral graft), and complex bulboprostatic anastomosis with or without inferior pubectomy. Excluded from this audit were urethral dilatations, suprapubic diversions, DVIU, hypospadias reconstruction.

All patients were operated in the exaggerated lithotomy under either spinal (no BMG required) or general anesthesia (BMG required). A detailed description of the individual procedures as done in our centre follows.

\section{Simple anastomotic urethroplasty}

For short $(1-3 \mathrm{~cm})$ strictures we used the simple anastomotic urethroplasty method, especially for bulbar urethra. Exposure was via a midline perineal incision. Depending on the anatomical site, the urethra was exposed after incising the dartos and bulbospongiosus layers. The strictured segment was excised completely; next, the healthy stumps were spatulated. Six interrupted sutures (Vicry1 4.0) were placed at $12,2,4,6,8$ and 10 o'clock positions. The bulbospongiosus and other layers were closed in a stepwise fashion, and no drains were left. A pressure dressing was applied and was removed 24 hours later.

\section{Buccal mucosal graft urethroplasty}

The urethra was exposed in standard fashion, as explained in the simple anastomotic urethroplasty method. Using the dorsal buccal mucosal graft (BMG) urethroplasty, the urethra was dissected off the corpora carvenosa by creating a sub-urethral widow and extending the dissection both proximally and distally. A dorsal urethrotomy was performed over a guide wire. Buccal mucosa graft was harvested from the right cheek, in standard fashion. The graft was defatted, fenestrated and quilted on the carvenosal bed with Vicryl 4.0 continuous sutures. The urethral edges were stitched to the quilted graft over a size 16 silicon catheter. Standard closure followed the simple anastomotic urethroplasty method. For ventral $\mathrm{BMG}$, a ventral urethrotomy was done, the graft stitched to the edges of the urethrotomy and the urethral sponge closed in two layers over a $16 \mathrm{Fr}$ silicon catheter.

\section{Non-transecting anastomotic bulbar urethroplasty}

The urethra was exposed in standard fashion as per the simple anastomotic method. A 2-cm longitudinal ventral urethrotomy was done over a guide wire. This was closed in a transverse Heineke-Mikulicz fashion. Standard skin closure followed.

\section{Complex bulboprostatic anastomosis}

One case of a pelvic floor urethral distraction defect (PFUDD) warranted this approach. A long, midline incision was made and the distal stump of urethra exposed in standard fashion. Transection was done at the most proximal point of the urethra that could admit a Fr 22 bougie. The proximal urethral impression was palpated and mobilized to access a segment of anastomosable healthy urethra, using a modified Hey Groove dilator (we curved a catheter introducer into a $\mathrm{C}$ shape and affixed a Foley catheter) advanced through the suprapubic cystostomy. It was necessary to incise the perineal 
membrane to facilitate prostatic urethra mobilization. We did not carry out inferior pubectomy. A tension-free bulboprostatic anastomosis was done at 12, 2, 4, 6, 8 and 10 o'clock positions with absorbable 4.0 sutures. Skin closure used standard methods.

Data were prospectively entered into a spreadsheet. The data included the following parameters: biodata, pre-operative adverse factors (high PFUDD, severe spongiofibrosis, watering can perineum, age $>70$ years, prolonged untreated obstructive uropathy), stricture etiology and characteristics (site, caliber, length, and recurrence), type of surgery (transecting anastomotic, non-transecting anastomotic, dorsal graft, ventral graft), hospital stay and complications (hematoma, site infection, recurrence, fistula, incontinence, ED). Successful short-term outcome was defined as subjective improvement of obstructive symptoms and not requiring a repeat procedure (catheter, dilatation, repeat urethroplasty) within 6 months (13).

\section{Results}

Between January and December 2017, 23 urethroplasties were carried out. The mean age of the patients was 52.5 years, and $83 \%$ presented primarily. The range of stricture length was 2-7 cm with a mean of $3 \mathrm{~cm}$. Nine strictures were inflammatory $(40 \%), 9$ were traumatic $(40 \%), 3$ were recurrent strictures and $2(9 \%)$ were unspecified. Most patients (63\%) were aged 30-50 years and presented posttrauma while $63 \%$ of those between $50-70$ years presented postinflammatory. The main clinical presentation was poor urine stream (10 patients, $43 \%$ ). The predominant location of the stricture was the bulbar urethra $(83 \%)$. Nine patients (40\%) had preoperative adverse risk factors.

Of the 23 urethroplasties, 13 were simple anastomosis (57\%), 5 were dorsal BMG urethroplasty (22\%), 2 were nontransecting anastomotic bulbar urethroplasty (NTABU), and 1 case each of ventral BMG urethroplasty, and Johanson's and Kulkarni's panurethroplasty (Table 1). The overall complication rate was 39\% (9 patients): 4 patients (17\%) had recurrence, 2 had fistula and 1 case each had persistent UTI, erectile dysfunction and periurethral abscess. Three of the four recurrences had undergone BMG urethroplasty. All cases of simple anastomosis had no recurrence.

\section{Discussion}

Urethral stricture is one of the leading causes worldwide of urine obstruction. A shift in the etiology from inflammatory to trauma occurred with the advent of effective antibiotics to treat sexually transmitted diseases (STD). The study sample was drawn from a rural region in Kenya where gonorrhea has been prevalent. Post-gonococcal urethritis sequelae are worsened by stigma associated with seeking care for STD. The finding of an equal number of trauma and inflammatory strictures was surprising. This finding could be explained

Table 1: Repair characteristics

\begin{tabular}{|c|c|c|}
\hline Location & $n$ & Repair type $(n)$ \\
\hline \multicolumn{3}{|l|}{ Trauma } \\
\hline Penile & 1 & Anastomotic \\
\hline Bulbar & 7 & $\begin{array}{l}\text { Anastomotic (5), NTABU, dorsal } \\
\text { BMG }\end{array}$ \\
\hline Bulbomembranous & 1 & $\begin{array}{l}\text { Complex bulboprostatic } \\
\text { anastomosis }\end{array}$ \\
\hline \multicolumn{3}{|l|}{ Inflammatory } \\
\hline Penile & 1 & Johanson's \\
\hline Bulbar & 7 & $\begin{array}{l}\text { Dorsal BMG (4), dentral BMG (1), } \\
\text { NTABU (2) }\end{array}$ \\
\hline Penobulbar & 1 & NTABU \\
\hline Penobulbar & 1 & Kulkarni's panurethroplasty \\
\hline Recurrent & 3 & Anastomotic \\
\hline Unspecified & 1 & Anastomotic \\
\hline
\end{tabular}

by the selection bias of patients most suitable for one-stage urethroplasty. Many other patients who present with urethral stricture disease are treated using urethral dilatation.

A predominance of bulbar strictures is well documented for both traumatic and, especially, inflammatory strictures. Infective bacteria predominantly affect the periurethral skene glands which are concentrated in the bulbar urethra, resulting in these strictures. In addition, the parts of a male urethra most vulnerable to trauma are the penobulbar and the bulbomembranous junctions. Our study concurred with prior studies from Africa and elsewhere $(14,15)$. Our mean urethral stricture length was $3 \mathrm{~cm}$, which was on the higher side. Again, this is a reflection of the selective bias for longer strictures which were not treated by simple dilatation. Dedicated urethroplasty centers have an average stricture length of 4-5 cm (16); this also likely reflects a selection bias towards referral of the most complex strictures.

The ease and reproducibility of simple anastomotic reconstruction makes it the mainstay of short urethral stricture surgery $(11,14,15,17)$. It is recommended mainly for sub-2-3cm strictures. This study achieved tension-free anastomosis of even up to $4-5 \mathrm{~cm}$ with reasonably good short-term results. Indeed, all $57 \%$ of the simple anastomosis done did not have complications. In a country with a dearth of specialized urological surgeons, this technique is encouraged for use by general surgeons and those with interest in urology to avoid long delays and unnecessary or costly referrals to urban centers. Our overall short-term 
success rate of $61 \%$ was in sharp contrast to a similar study in Tanzania that showed a $90.8 \%$ one-year success rate (18). The Tanzanian study further found $100 \%$ success in substitution urethroplasty. The study site was a large tertiary hospital with better equipment and several specialists, and these impressive results are not surprising.

A significant number of complications were noted in our study with the substitution urethroplasties (overall 39\%); 7 of the cases were major complications. As one of the aims of this interim study was to assess the safety of complex urethral surgery in a new urethroplasty centre, these complications were not unexpected. A 62-month study done in Chicago, Illinois, found an 18\% long-term recurrence rate of anastomotic urethroplasty compared with $31 \%$ recurrence with substitution urethroplasty (19). The general long-term recurrence ranges from $1.2 \%$ to $14 \%$ for anastomotic urethroplasty and up to $58 \%$ for substitution urethroplasty $(20,21)$. Being a new centre of reconstruction, these high figures serve as a wake-up call to review our systems and especially patient and technique selection.

The major weakness of our study was the short-term followup. At one year, no robust inferences can be made on any of the major outcome measures for urethroplasty. This interim study was a safety and effectiveness audit not designed for long-term outcome. In addition, our centre was unable to proceed with further patient follow-up due to circumstances beyond our control.

\section{Conclusion}

Complex urethroplasty has a clinically significant complication rate, but our recurrence rate for simple urethroplasty is low. A high recurrence was noted in substitution urethroplasties, suggesting the need for careful patient selection, an improvement in technique and possible early referral of these cases to specialized centers.

\section{References}

1. Mundy AR, Andrich DE. Urethral strictures. BJU Int. 2011; 107(1):6-26.

2. Eshiobo I. A 10-year review of urethral stricture management in Irrua, Nigeria. 2017; 23(2):119-24.

3. Balbay MD, Ergen A, Sahin A, et. al. Development of urethral stricture after transurethral prostatectomy: A retrospective study. Int Urol Nephrol. 1992; 24(1):49-53.

4. Rassweiler J, Teber D, Kuntz R, Hofmann R. Complications of transurethral resection of the prostate (TURP) - Incidence, management, and prevention. Eur Urol. 2006; 50(5):969-79.
5. Alwaal A, Blaschko SD, McAninch JW, Breyer BN. Epidemiology of urethral strictures. Transl Androl Urol. 2014; 3(2):209-13.

6. Osoba AO, Alausa O. Gonococcal urethral stricture and watering can perineum. Br J Vener Dis. 1976; 52(6):387-93.

7. McMillan A, Pakianathan M, Mao JH, MacIntyre CCA. Urethral stricture and urethritis in men in Scotland. Sex Transm Infect. 1994; 70(6):403-5.

8. Kamande L, Owilla F. A review on management procedures for urethral stricture disease at Kenyatta National Hospital. Unpublished dissertation, University of Nairobi, 2003.

9. Oliech JS. One-stage repair of urethral strictures at the Kenyatta National Hospital (1978-1984). East Afr Med J. 1988; 65(1):39-47.

10. Adwok JA. Urethral strictures at the Kenyatta National Hospital. East Afr Med J. 1986; 63(3):175-81.

11. Dakum NK, Ramyil VM, Amu CO. Outcome of urethroplasty for urethral stricture at Jos University Teaching Hospital. Niger J Clin Pract. 2008; 11(4):300-4.

12. Kulkarni S, Kulkarni J, Surana S, Joshi PM. Management of panurethralstricture. UrolClin North Am. 2017; 44(1):67-75.

13. Jackson MJ, Chaudhury I, Mangera A, Brett A, Watkin N, Chapple CR, et al. A prospective patient-centred evaluation of urethroplasty for anterior urethral stricture using a validated patient-reported outcome measure. Eur Urol. 2013; 64(5):77782.

14. Hussain A, Pansota MS, Rasool M, Tabassum SA, Ahmad I, Saleem MS. Outcome of end-to-end urethroplasty in posttraumatic stricture of posterior urethra. J Coll Physicians Surg Pakistan. 2013; 23(4):272-5.

15. Eziyi AK, Olajide AO, Etonyeaku AC, Ojewuyi OO, Eziyi JAE, Adeyanju T, et al. One-stage urethroplasty for urethral strictures at the Ladoke Akintola University of Technology Teaching Hospital, Osogbo, South Western Nigeria. World J Surg. 2013; 37(11):2529-33.

16. Kinnaird AS, Levine MA, Ambati D, Zorn JD, Rourke KF. Stricture length and etiology as preoperative independent predictors of recurrence after urethroplasty: A multivariate analysis of 604 urethroplasties. J Can Urol Assoc. 2014; 8(56):296-300.

17. Aghaji AE, Odoemene CA. One-stage urethroplasty for strictures: Nigerian experience. Int J Urol. 2001; 8(7):380-5.

18. Musau P, Mteta AK. Urethral strictures in a tertiary care hospital in Tanzania. East Afr Med J. 2009; 86(1):3-6.

19. Han JS, Liu J, Hofer MD, Fuchs A, Chi A, Stein D, et al. Risk of urethral stricture recurrence increases over time after urethroplasty. Int J Urol. 2015; 22(7):695-9.

20. Andrich DE, Dunglison N, Greenwell TJ, Mundy AR. The long-term results of urethroplasty. J Urol. 2003; 170(1):90-2.

21. Eltahawy EA, Virasoro R, Schlossberg SM, McCammon KA JG. Long-term follow-up for excision and primary anastomosis for anterior urethral strictures. J Urol. 2007; 177:1803-6. 